\title{
Postsaccadic Activities in the Posterior Parietal Cortex of Primates Are Influenced by both Eye Movement Vectors and Eye Position
}

\author{
Aldo Genovesio, Emiliano Brunamonti, Maria Assunta Giusti, and Stefano Ferraina \\ Department of Human Physiology and Pharmacology, University "La Sapienza," 00185 Rome, Italy
}

\begin{abstract}
Primates explore their visual environment by redirecting the gaze to objects of interest by alternating eye movements and periods of steady fixation. During this task, the fixation point changes frequently in depth. Therefore, the representation of object location based on retinal disparity requires frequent updating. Neural activity was recorded in the lateral intraparietal (LIP) area while monkeys performed saccades between targets in different depths. We report that in the early postsaccadic period, posterior parietal neurons continue to encode the difference in depth between fixation point and targets. About one-third of these neurons are, during the same period, modulated by eye position in depth as well. In the late postsaccadic period, the influence of the previous movement vector dissipates, and parietal neurons are modulated only by the new fixation distance. This result suggests that the postsaccadic activity of area LIP contributes to the dynamic representation of the visual space, and it is compatible with the presence of both a vector subtraction computation and eye-position-dependent gain fields.
\end{abstract}

Key words: posterior parietal cortex; eye movements; distance; monkeys; space remapping; depth

\section{Introduction}

Primates explore their three-dimensional (3-D) visual environment by redirecting the gaze to objects of interest through eye movements followed by periods of fixation. In doing so, the fixation point (FP) changes frequently in depth. There is ample evidence that vision is suppressed or reduced during saccades (Bridgeman et al., 1975; Stark et al., 1976; Schlag and Schlag-Rey 1995; Morrone et al., 1997). Therefore, the 3-D representation of object location based on retinal disparity and eye position signals requires frequent updating, after each saccade.

Lateral intraparietal (LIP) area neurons are active during the visuomotor events preceding the initiation of saccadic eye movements (Gnadt and Andersen, 1988) and integrate retinal and extraretinal signals (Andersen et al., 1990; Bremmer et al., 1997). The visuomotor-related activities of LIP neurons are tuned in depth (Gnadt and Mays, 1995; Ferraina et al., 2002; Genovesio and Ferraina, 2004) and contribute to the formation of an egocentric representation of the extrapersonal space. They display an integration of retinal disparity and vergence angle in the form of a partial shift of disparity tuning with changes in fixation distance (Genovesio and Ferraina, 2004). LIP neurons also participate in the remapping of visual space occurring around the time of the saccade (Duhamel et al., 1992a). The remapping mechanism is

Received June 10, 2006; revised Feb. 23, 2007; accepted Feb. 23, 2007.

This work was supported by Ministero dell'Università e della Ricerca Grant 2005051741, partially by Italian National Institute of Health Grant 530/F4/1, and by University of Rome "La Sapienza" Grant C26G031031. We are grateful to R. Caminiti for support throughout this research.

Correspondence should be addressed to Dr. Stefano Ferraina, Department of Human Physiology and Pharmacology, University “La Sapienza,” Piazzale Aldo Moro 5, 00185 Rome, Italy. E-mail: stefano.ferraina@uniroma1.it.

D0I:10.1523/JNEUROSC1.5415-06.2007

Copyright $\odot 2007$ Society for Neuroscience $\quad$ 0270-6474/07/273268-06\$15.00/0 thought to be important because it offers the possibility to maintain an accurate representation of the visual world soon after a saccade.

Many LIP neurons discharge in the postsaccadic epoch (Barash et al., 1991). This activity may reflect a variety of signals, including the current eye position. However, this activity could also reflect the previous eye movement vector and/or previous eye position. Because the eye movements made in most oculomotor tasks have different end points, these possibilities have not been yet tested.

In this report, we will show that in the postsaccadic period, the neural activity is influenced conjunctively by the new eye position and by the previous eye movement vector. The coexistence of these signals takes place after movement completion, when the information remapped needs to be integrated with actual sensory feedback (Heide et al., 2001; van Donkelaar and Muri, 2002; Bellebaum et al., 2005). A brief report has appeared previously (Ferraina et al., 2005).

\section{Materials and Methods}

Two rhesus monkeys (Macaca mulatta) were studied using general procedures described recently (Genovesio and Ferraina, 2004). Monkeys sat in a primate chair facing two robot arms used to place a red light-emitting diode (LED) ( $<0.5^{\circ}$ diameter spot) at different distances from the eyes. The workspace was formed by eight virtual isovergence planes (IVG) (Fig. 1a) passing through the rotational centers of the two eyes, calculated taking into account the interocular distances $(36$ and $34 \mathrm{~mm}$, respectively) of the two monkeys, and separated in depth by steps of $1^{\circ}$ of vergence angle. The closest isovergence plane corresponded to $13^{\circ}$ and the farther to $6^{\circ}$ of vergence angle.

To determine the locus of maximal neural activity in the response field, during the preliminary exploration of the lateral bank of the in- 

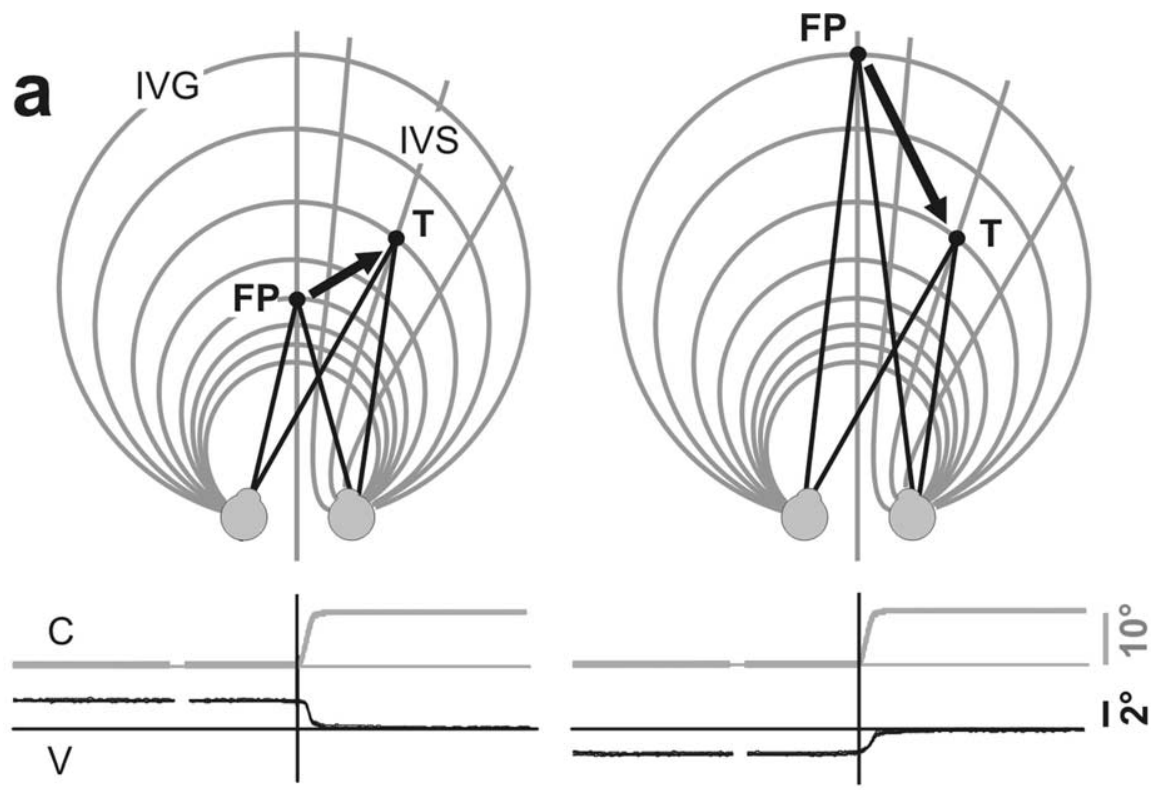

b
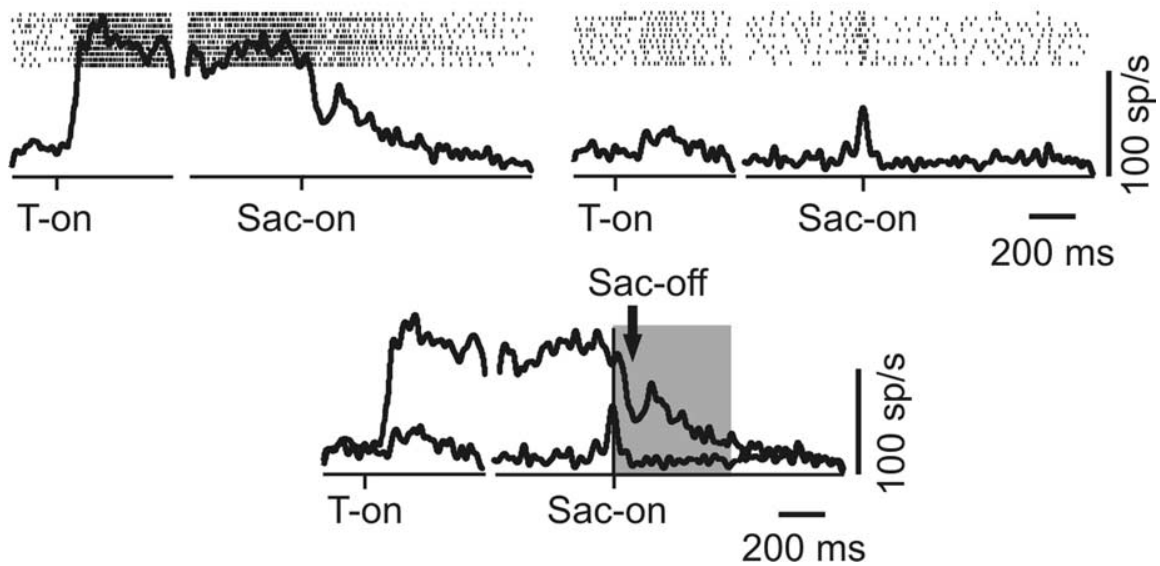

Figure 1. Schematic of the task and example of effect of the starting position on the postsaccadic activity. $\boldsymbol{a}$, Top, Identica target $(T)$ locations are foveated by saccadic eye movements starting from different fixation distances (FPs). Every movement requires a combination of vergence and version components. IVG and IVS are two-dimensional representations of isovergence and isoversion loci, respectively. Bottom, The conjugate ( $C$; gray lines) and the vergence (V; black lines) eye position are shown as a function of time. The alignment is at the beginning of the saccadic eye movement (vertical line). Zero lines and calibration bars are shown for both movements. $\boldsymbol{b}$, Top, Rasters and spike density functions of neural data aligned to target presentation (T-on) and beginning of eye movements ( $\mathrm{Sac}-\mathrm{On}$ ). Bottom, The spike density functions are overlapped. The shaded region indicates $500 \mathrm{~ms}$ epoch after Sac-on. The approximate end of movement (Sac-off) is indicated. sp/s, Spikes per second.

targets, were annulled after movement completion. For the majority of the neurons reported in the present study, monkeys were required to start the movement either from the closer $\left(13^{\circ}\right.$ of vergence angle) or the farther $\left(6^{\circ}\right.$ of vergence angle) fixation plane and to move toward targets located at one of eight different depths. However, these data were selected from a larger dataset in which the movements started from all eight of the starting positions (FPs) (see also Genovesio and Ferraina, 2004).

Area LIP was identified physiologically in the IPS by the abundance of neurons with visual responses, as well as saccade-related activity. Subsequent standard histological procedures confirmed the location of the recordings in the middle third of the lateral bank of the IPS.

Eye position was monitored by using a scleral search coil in each eye. Calibration was obtained by requiring the monkeys to fixate sequentially on five LEDs, one central and the others positioned to form an angle of -10 and $+10^{\circ}$ both in the vertical and in the horizontal axis of the IVG corresponding to $10^{\circ}$ of vergence angle. Calibration and control were separated for each eye. During calibration, the center LED was aligned with the eye that was being calibrated while the other eye was covered. Movement starting and ending times were computed off-line by using a threshold speed of $25^{\circ} \mathrm{s}$. An eye movement was considered successful if each eye landed within a $3^{\circ}$ (diameter) spherical window centered on the target. More than $85 \%$ of the trials resulted in a vergence error of $<0.3^{\circ}$. Trials with vergence errors $>0.5^{\circ}$ were excluded from additional analysis. Animal care, housing, and surgical procedures were in accordance with European guidelines (European Community Council Directive 86/ 609/ECC) and with the Italian national law (DL $116 / 92$ ) on the use of animals in research.

For data analysis, rasters of neuronal discharges and time-varying spike density functions (Richmond et al., 1987) were aligned to specific events of the behavioral paradigm. Raw spike counts were used to measure neuronal activity during successive epochs of the tasks. To generate the spike density function, a Gaussian pulse ( $\sigma=10 \mathrm{~ms}$ ) was substituted to each spike, and then all Gaussians were summed to produce a function continuous in time.

\section{Results}

traparietal sulcus (IPS), eye movements were made to a two-dimensional array of targets located at different elevations and eccentricities along the isovergence surface (IVS) corresponding to $10^{\circ}$ of vergence angle. Target eccentricity was tested from 10 to $20^{\circ}$; target elevation was tested from -20 to $20^{\circ}$ in steps of $10^{\circ}$. Each trial started with the presentation of a central FP (Fig. 1a) and, after 500-800 ms of fixation, a peripheral visual target appeared on the predetermined locus of maximal neural activity and remained on until the end of the trial. The depth of the FP varied among isovergence surfaces and from trial to trial. Within $500 \mathrm{~ms}$ of the disappearance of the FP (Go signal), after an 800-1200 ms delay period, the monkey was required to make a saccade to the target and to maintain that eye position for a variable time (1000-1200 ms). For each trial, targets and FPs were arranged always at identical retinal eccentricity (IVS) (Fig. 1a) but at different relative positions in depth to obtain either positive or negative differences between the initial and final vergence angles. These differences, corresponding to the retinal disparity of the
As described previously (Barash et al., 1991; Ferraina et al., 2002; Genovesio and Ferraina, 2004; Gnadt and Andersen, 1988), during the generation of delayed saccadic eye movements, LIP neurons display a visual-related response that is maintained for the duration of the delay period and that often increases before saccade generation. We have previously reported that these features are influenced by the retinal disparity of the target (Genovesio and Ferraina, 2004) for saccadic eye movements in depth.

For the current analysis we selected, from the previously published dataset (Genovesio and Ferraina, 2004), the neurons (120 of 171 ) with significant activity ( $t$ test; $p<0.05$ ) in the postsaccadic epoch (500 ms interval after saccade completion) compared with the control period (from 500 to 200 ms before target presentation). When there was a significant difference for at least three 
different movements in depth, we deemed the neuron as modulated and included it on additional analysis.

Figure 1 shows an example neuron studied in the course of the generation of two different classes of eye movements directed to the identical position in retinal eccentricity $\left(10^{\circ}\right.$ to the right of the FP) and depth (fixation distance corresponding to $8^{\circ}$ of vergence angle) but starting from two different depths (10 and $6^{\circ}$, respectively). The two illustrated disjunctive eye movements required the same change $\left(2^{\circ}\right)$ of vergence angle of opposite sign. The conjugate component of the movements was identical. The neural modulation (Fig. 1b) reflected the difference in depth between FP and targets since the presentation of the target. Thereafter, the influence of the previous movement lasted for $\sim 500 \mathrm{~ms}$ after the movement (Fig. 1b, shaded area).

We limited the analysis of the postsaccadic activity to the data obtained combining the movements from only two starting fixation distances ( 6 and $13^{\circ}$ of vergence angle) (Fig. 2, top) to eight target locations. Figure 2 shows the neural activity of two different classes of neurons. Both neurons display during the postsaccadic period a tuning for the different positions in depth with a preference for the nearest locations. Moreover, the neuron on the left is additionally modulated in the first $250 \mathrm{~ms}$ (gray area) by the initial eye position.

To identify these two main types of neurons a sliding window of $100 \mathrm{~ms}$ width, displaced for $1000 \mathrm{~ms}$ in $20 \mathrm{~ms}$ steps and starting at the time of the end of the saccade, was used to test (two-way ANOVA) whether the average neural activity of each bin was either significantly modulated ( $p<0.05$ ) by the initial fixation position (near or far) and/or by the eye final position. For each factor, when the modulation lasted for at least five significant tests in a row, we deemed the cell modulated. We identified two main groups of cells: a first group (42 of 120; $35 \%$ ) displayed a modulation in the postsaccadic activity influenced by the initial eye position, as in the example of Figure 2 (left); a second group (21 of $120 ; 18 \%$ ) displayed a modulation in the postsaccadic activity influenced only by the final eye position, as in the example of Figure 2 (right). The modulation for the initial eye position emerged only in the early phases of the postsaccadic epoch, mainly (30 of 42; 71\%) within $320 \mathrm{~ms}$ after saccade completion and never later than $400 \mathrm{~ms}$. For approximately half ( 24 of $42 ; 57 \%$ ) of these neurons, the modulation was evident since the moment of saccade end; for the remaining neurons, the modulation emerged only after $80 \mathrm{~ms}$ from saccade end. A subgroup of neurons modulated by the initial eye

Sac-on 1. sp/s, Spikes per second.
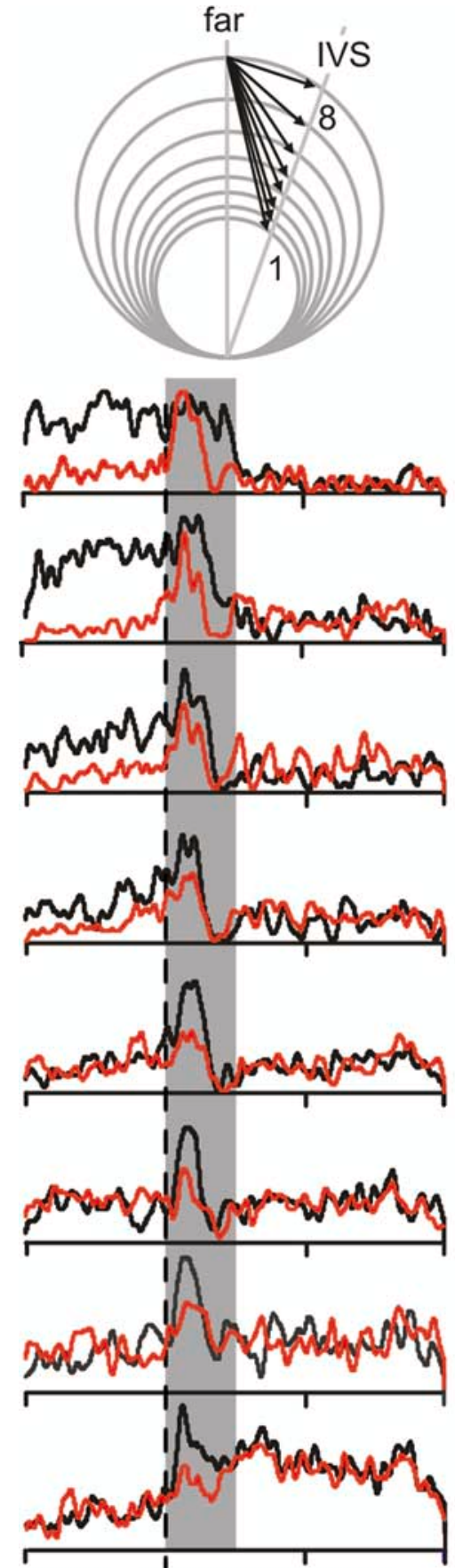

Figure 2. Postsaccadic activity in two different neurons. Spike density functions of neural data aligned to beginning of eye movements (Sac-on). Data are shown for far (black lines) and near (red lines) origin of the movements directed to eight identical target locations (1-8). Left, Neuron additionally influenced by the initial position in depth during the early postsaccadic period (gray area). Right, Neuron influenced only by the final position of the target in depth. Other conventions and symbols as in Figure

position was additionally sensitive to the final eye position (19 of $42 ; 45 \%$ ). In six ( 6 of $19 ; 32 \%$ ) of them, the sensitivity for the final position was maintained for all of the postsaccadic analyzed period; in seven neurons ( 7 of 19; 37\%), it emerged only after the sensitivity for the initial position was lost. In the remaining six cells, the influence of the final eye position appeared time locked to the sensitivity for the previous positions. Therefore, in approximately one-third $(12$ of $42 ; 28.6 \%)$ of the neurons influenced by 

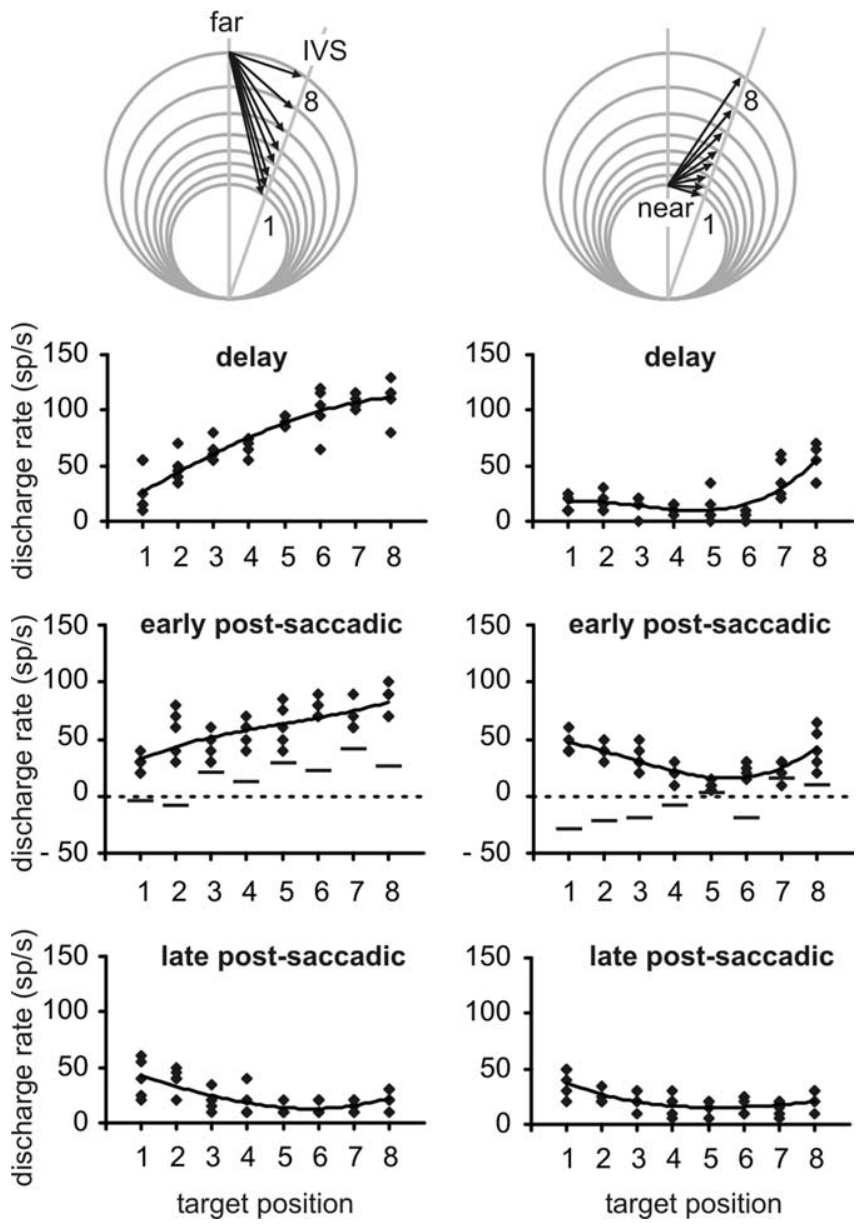

Figure 3. Effect of the target position on the neural modulation for the three epochs analyzed. Data are from an example neuron. Tuning curves are obtained for the different epochs by fitting the data, obtained in each single trial (five total), to a cubic model. Left and right columns show, respectively, data obtained for far and near origin of the movements. Horizontal lines in the middle panels indicate the neural activity difference between early postsaccadic and delay epochs. sp/s, Spikes per second.

the initial position of the movement, there was a concurrent modulation for the final position.

We were interested in whether the activity observed during the period of target fixation would be related to the activity during the preparation of movement. Therefore, in the 42 neurons influenced by the initial eye position, we performed an additional partial correlation analysis, using as a control variable the target position, for three selected epochs: the delay (300 ms preceding the Go signal), the early postsaccadic (from 100 to $300 \mathrm{~ms}$ after saccadic end), and the late postsaccadic (from 500 to $700 \mathrm{~ms}$ ) epochs. The choice of these temporal windows originated from the results of the two-way ANOVA analysis as well as for obtaining activities not confounded, as much as possible, by concurrent signals. Therefore, the end of the postsaccadic period was excluded because of the possibility of having a preparatory activity for the return movement to the next FP. Figure 3 shows, for a typical neuron, the activity tuning for each epoch separated for the two main classes of movement. This neuron was modulated during the delay epoch (top) by the different location in depth of the targets. During the early postsaccadic period (middle), the neuron was modulated both by the target position and by the initial eye position (near or far). During the late postsaccadic epoch (bottom), the tuning for the target position in depth was

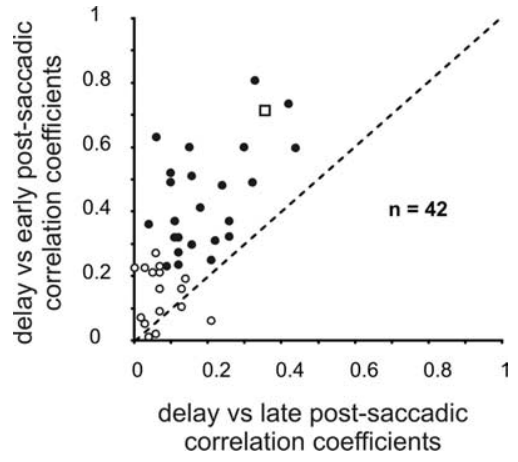

Figure 4. The early postsaccadic activity is correlated with the activity in the delay epoch. Scatter plot of the correlation coefficients. Filled symbols correspond to statistically significant $(p<0.05)$ correlation coefficients between early postsaccadic and delay epochs. The empty square corresponds to values obtained for the neuron of Figure 3 . The equality line is shown.

not affected anymore by the eye movement starting positions. The activity tuning in the delay and in the early postsaccadic epochs appeared very similar, during both converging and diverging disjunctive eye movements. The lines in the middle panels indicate the difference in activities between the early postsaccadic and the delay epochs. In this neuron and in most of the other neurons, the difference in activity between delay and early postsaccadic epochs changes sign depending on the target position.

Figure 4 contrasts the absolute values of the correlation coefficients obtained for the delay and the early postsaccadic period correlation ( $y$ axes) versus the absolute values of the correlation coefficients obtained for the delay and the late postsaccadic correlation ( $x$ axes). Most of the neurons showed a higher correlation of the delay activity with the early postsaccadic activity than with the late postsaccadic activity. The empty square symbol indicates the results obtained for the neuron in Figure 3. In summary, most of the neurons influenced by the initial eye position in depth during the early postsaccadic epoch showed a correlation of their neural activity with the neural activity observed at the time of movement preparation.

\section{Discussion}

The present study is the first to show that individual LIP neurons maintain a signal related to the preceding eye movement vector after saccade completion. This signal, when present, is correlated with the eye motor error signal (difference in depth between FP and targets) observed before saccade generation. Having this signal available, after the saccade completion, could play an important role in maintaining the three-dimensional perception stability.

Retinal images of external objects are displaced each time the eyes move. Nevertheless, we are able to accurately determine the egocentric location of targets and to produce accurate movements to these targets. This implies that the brain monitors the metrics of each eye movement as the difference between the current and the preceding position of the eye in the orbit.

The importance of extraretinal signals for space coding is not new for area LIP. At the single cell level, the integration of visual and visuomotor signals in the form of gain field (Andersen et al., 1990; Bremmer et al., 1997) is believed to be used to compute the position of visual targets in head-centered coordinates (Zipser and Andersen 1988).

Retinal disparity signals have been described in area LIP (Gnadt and Mays, 1995; Ferraina et al., 2002; Genovesio and Ferraina, 2004), and the integration of retinal disparity and fixa- 
tion distance information can be used to determine 3-D egocentric distance of an object (Genovesio and Ferraina, 2004).

Patients with posterior parietal cortex (PPC) lesions (Duhamel et al., 1992b) are impaired in the performance of the double-step paradigm. In this task, the position of two sequential briefly flashed targets is presented during the latency of the first saccade, and subjects are instructed to make two saccades in sequence to the locations at which the peripheral targets have been presented. In such tasks, when the first saccade is directed to the contralesional hemifield, the second saccade is dysmetric. Therefore, after the first saccade, the oculomotor system cannot use retinal error signals to guide the movement toward the second target and needs access to extraretinal information about the metrics of the eye movement. Goldberg and Bruce (1990) pointed out that the computation necessary to execute the second saccade in the double-step task can be described as a vector subtraction, in which the second saccade is the retinal vector of the second target minus the vector of the first saccade.

LIP neurons anticipate the retinal consequences of a programmed movement by responding to visual stimuli appearing in their future receptive field (Duhamel et al., 1992a). Neurons with similar properties have been described in frontal eye field (FEF) (Umeno and Goldberg, 1997) and superior colliculus (Walker et al., 2005). Furthermore, it has been shown that the superior colliculus relays corollary discharge signals to FEF via the mediodorsal thalamus (Wurtz and Sommer, 2004), and FEF in turn is functionally coupled with area LIP (Ferraina et al., 2002). This distributed network is thought to be a prerequisite for the implementation of a vector subtraction computation. Our data show that in the early phases of the fixation period after a saccadic eye movement, the neural activity of LIP neurons is influenced by the previous eye movement vector. In these neurons, the effect of the oculomotor plan is not cancelled instantaneously after saccade completion but persists longer. A representation of the previous eye movement vector provides the oculomotor network with a signal that could be used to maintain remapped visual information until the moment in which it could be integrated with the actual sensory feedback.

In the PPC, a similar observation has been reported for V6a neurons (Nakamura et al., 1999) during bidimensional saccadic eye movements. In V6a, the majority of the cells tested responded in relation to the direction of these saccades that preceded fixation, although their activity was more influenced by eye position than by previous saccade direction.

The time course of our observation is in agreement with recent reports in humans. By interfering with parietal functions through TMS (van Donkelaar and Muri, 2002), as well as using EEG studies (Bellebaum et al., 2005), it was possible to conclude that remapping, in the contest of the double-step saccade paradigm, takes place in the early phases of the postsaccadic epoch after the first saccade. Postsaccadic evoked response components, recorded in the human's parietal cortex have been interpreted as the basis of an incoming efference copy signal (Anagnostou et al., 2000). Additional insight on the continuation of the remapping process in the postsaccadic period comes from the experiment of Lappe et al. (2000), in which a compression of the presaccadic space can still be caused by visual references immediately after a saccade. This result suggests that the visual remapping process is not instantaneous but continues during the period of acquisition of postsaccadic image information.

We found that $45 \%$ of the neurons selective for the previous eye movement vector were modulated by eye position as well.
Moreover, most of them were simultaneously modulated for both signals. Whereas information about the previous eye movement vector may be used to maintain a memory based representation of visual objects, the eye position information may be used to encode the visual information received in real time in a distributed head-centered system of coordinate. The two proposed mechanisms, remapping by eye vector subtraction and use of eye position signals for generating a distributed head-centered representation of the visual space, are not necessarily mutually exclusive; in fact, both eye position signals and eye displacements signals could be used for coordinate transformations. One possibility is that LIP may be involved in integrating in the postsaccadic period the visual information from two sources, one obtained through remapping and the other based on the peripheral view of the target obtained after an eye movement.

Whereas the remapping is based on the use of a copy of the eye motor commands, the representation in head-centered coordinate of the visual space relies on eye position information. The presence of both signals in the postsaccadic period may reflect the coexistence of the initial remapped information with postsaccadic visual information. Vaziri et al. (2006) have shown that integration of information from these two sources is useful to produce a more precise estimate of the visual targets for reaching than is possible from either source alone.

In summary, the results of the present experiment, even with the limitations of being a single-saccade task, suggest a new role for the postsaccadic activity in area LIP in integrating different representations of the visual space after the end of the movement. Other areas of the PPC and cortical or subcortical regions could have a similar role as well. Furthermore, the computation shown is based on binocular extraretinal signals, whereas most of the studies used a bidimensional approach. Additional studies, of the physiological mechanisms subtending space stability, are required to distinguish the relative contribution of eye displacement and eye position signals in the contest of the updating process. Future studies need to address this issue using both singleand double-saccade trials as well as saccades both in depth and in the bidimensional plane.

\section{References}

Anagnostou E, Kleiser R, Skrandies W (2000) Electrophysiological correlates of human intrasaccadic processing. Exp Brain Res 130:177-187.

Andersen RA, Bracewell RM, Barash S, Gnadt JW, Fogassi L (1990) Eye position effects on visual, memory, and saccade-related activity in areas LIP and 7A of macaque. J Neurosci 10:1176-1196.

Barash S, Bracewell RM, Fogassi L, Gnadt JW, Andersen RA (1991) Saccaderelated activity in the lateral intraparietal area. I. Temporal properties; comparison with area 7a. J Neurophysiol 66:1095-1108.

Bellebaum C, Hoffmann KP, Daum I (2005) Post-saccadic updating of visual space in the posterior parietal cortex in humans. Behav Brain Res 163:194-203.

Bremmer F, Distler C, Hoffmann KP (1997) Eye position effects in monkey cortex. II. Pursuit- and fixation-related activity in posterior parietal areas LIP and 7A. J Neurophysiol 77:962-977.

Bridgeman B, Hendry D, Stark L (1975) Failure to detect displacement of the visual world during saccadic eye movements. Vision Res 15:719-722.

Duhamel J-R, Colby CL, Goldberg ME (1992a) The updating of the representation of visual space in parietal cortex by intended eye movements. Science 255:90-92.

Duhamel J-R, Goldberg ME, Fitzgibbon EJ, Sirigu A, Grafman J (1992b) Saccadic dysmetria in a patient with a right frontoparietal lesion. The importance of corollary discharge for accurate spatial behaviour. Brain 115:1387-1402.

Ferraina S, Paré M, Wurtz RH (2002) Comparison of cortico-cortical and cortico-collicular signals for the generation of saccadic eye movements. J Neurophysiol 87:845-858. 
Ferraina S, Genovesio A, Brunamonti E (2005) Postsaccadic contribution to spatial stability in the lateral intraparietal area. Soc Neurosci Abstr 31:590.5.

Genovesio A, Ferraina S (2004) Integration of retinal disparity and fixationdistance related signals toward an egocentric coding of distance in the posterior parietal cortex of primates. J Neurophysiol 91:2670-2684.

Gnadt JW, Andersen RA (1988) Memory related motor planning activity in posterior parietal cortex of macaque. Exp Brain Res 70:216-220.

Gnadt JW, Mays LE (1995) Neurons in monkey parietal LIP are tuned for eye-movement parameters in three-dimensional space. J Neurophysiol 73:280-297.

Goldberg ME, Bruce CJ (1990) Primate frontal eye fields. III. Maintenance of a spatially accurate saccade signal. J Neurophysiol 64:489-508.

Heide W, Binkofski F, Seitz RJ, Posse S, Nitschke MF, Freund HJ, Kompf D (2001) Activation of frontoparietal cortices during memorized triplestep sequences of saccadic eye movements: an fMRI study. Eur J Neurosci 6:1177-1189.

Lappe M, Awater H, Krekelberg B (2000) Postsaccadic visual references generate presaccadic compression of space. Nature 403:892-895.

Morrone MC, Ross J, Burr DC (1997) Apparent position of visual targets during real and simulated saccadic eye movements. J Neurosci 17:7941-7953.

Nakamura K, Chung HH, Graziano MS, Gross CG (1999) Dynamic representation of eye position in the parieto-occipital sulcus. J Neurophysiol $81: 2374-2385$.
Richmond BJ, Optican LM, Podell M, Spitzer H (1987) Temporal encoding of two-dimensional patterns by single units in primate inferior temporal cortex. I. Response characteristics. J Neurophysiol 57:132-146.

Schlag J, Schlag-Rey M (1995) Illusory localization of stimuli flashed in the dark before saccades. Vision Res 35:2347-2357.

Stark L, Kong R, Schwartz S, Bridgeman DHB (1976) Saccadic suppression of image displacement. Vision Res 16:1185-1187.

Umeno MM, Goldberg ME (1997) Spatial processing in the monkey frontal eye field. I. Predictive visual responses. J Neurophysiol 78:1373-1383.

van Donkelaar P, Muri R (2002) Craniotopic updating of visual space across saccades in the human posterior parietal cortex. Proc Biol Sci 269:735-739.

Vaziri S, Diedrichsen J, Shadmehr R (2006) Why does the brain predict sensory consequences of oculomotor commands? Optimal integration of the predicted and the actual sensory feedback. J Neurosci 26:4188-4197.

Walker MF, FitzGibbon EJ, Goldberg ME (2005) Neurons in the monkey superior colliculus predict the result of impending saccadic eye movements. J Neurophysiol 73:1988-2003.

Wurtz RH, Sommer MA (2004) Identifying corollary discharges for movement in the primate brain. Prog Brain Res 144:47-60.

Zipser D, Andersen RA (1988) A back-propagation programmed network that simulates response properties of a subset of posterior parietal neurons. Nature 331:679-684. 